\section{Crystal structure of 2-\{[(2-chlorophenyl)- imino]methyl\}phenol}

\author{
Matheswaran Saranya, ${ }^{\mathrm{a}}$ Annamalai Subashini, ${ }^{\mathrm{a} *}$ \\ Chidambaram Arunagiri ${ }^{b}$ and Packianathan Thomas \\ Muthiah $^{\mathrm{c}}$
}

apG \& Research Department of Chemistry, Seethalakshmi Ramaswami College,

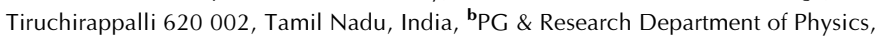
Government Arts College, Ariyalur 621 713, Tamil Nadu, India, and ' School of Chemistry, Bharathidasan University, Tiruchirappalli 620 024, Tamil Nadu, India.

*Correspondence e-mail: asubashini2k4@yahoo.co.in

Received 6 December 2014; accepted 9 December 2014

Edited by W. T. A. Harrison, University of Aberdeen, Scotland

In the title compound, $\mathrm{C}_{13} \mathrm{H}_{10} \mathrm{ClNO}$, the dihedral angle between the planes of the aromatic rings is $51.42(9)^{\circ}$ and an intramolecular $\mathrm{O}-\mathrm{H} \cdots \mathrm{N}$ hydrogen bond closes an $S(6)$ ring. The $\mathrm{Cl}$ atom and the $\mathrm{N}$ atom are syn. No directional interactions beyond van der Waals contacts are observed in the crystal.

Keywords: crystal structure; 2-\{[(2-chlorophenyl)imino]methyl\}phenol; Schiff base; van der Waals contacts.

CCDC reference: 1038374

\section{Related literature}

For related structures recently reported by us and background to Schiff bases, see: Arunagiri et al. $(2013 a, b)$. For a related structure, see: Chumakov et al. (2005).

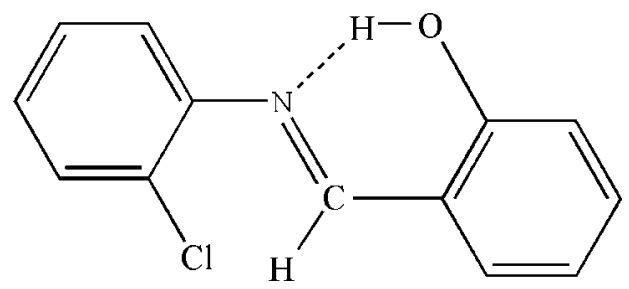

\section{Experimental}

\subsection{Crystal data}

$\mathrm{C}_{13} \mathrm{H}_{10} \mathrm{CINO}$

$M_{r}=231.67$

Orthorhombic, $P 2_{1} 2_{1} 2_{1}$

$a=6.8591(2) \AA$

$b=12.1829$ (4) $\AA$

$c=13.5405(5) \AA$

\subsection{Data collection}

Bruker Kappa APEXII CCD diffractometer 6509 measured reflections

$V=1131.50(6) \AA^{3}$

$Z=4$

Mo $K \alpha$ radiation

$\mu=0.31 \mathrm{~mm}^{-1}$

$T=293 \mathrm{~K}$

$0.30 \times 0.25 \times 0.20 \mathrm{~mm}$

\subsection{Refinement}

$R\left[F^{2}>2 \sigma\left(F^{2}\right)\right]=0.034$

$w R\left(F^{2}\right)=0.097$

$S=1.06$

2744 reflections

146 parameters

$\mathrm{H}$-atom parameters constrained

2744 independent reflections 2315 reflections with $I>2 \sigma(I)$ $R_{\text {int }}=0.017$

Table 1

Hydrogen-bond geometry $\left(\AA,{ }^{\circ}\right)$.

\begin{tabular}{lllll}
\hline$D-\mathrm{H} \cdots A$ & $D-\mathrm{H}$ & $\mathrm{H} \cdots A$ & $D \cdots A$ & $D-\mathrm{H} \cdots A$ \\
\hline $\mathrm{O} 1-\mathrm{H} 1 \cdots \mathrm{N} 1$ & 0.82 & 1.88 & $2.611(2)$ & 147 \\
\hline
\end{tabular}

Data collection: APEX2 (Bruker, 2008); cell refinement: SAINT (Bruker, 2008); data reduction: $S A I N T$; program(s) used to solve structure: SHELXS97 (Sheldrick, 2008); program(s) used to refine structure: SHELXL97 (Sheldrick, 2008); molecular graphics: PLATON (Spek, 2009); software used to prepare material for publication: PLATON.

\section{Acknowledgements}

MS thanks Collegiate Education Chennai, Tamil Nadu, for financial support (College Research Student Fellowship; reference No. 28696/K2/12).

Supporting information for this paper is available from the IUCr electronic archives (Reference: HB7335).

\section{References}

Arunagiri, C., Subashini, A., Saranya, M. \& Thomas Muthiah, P. (2013a). Elixir Org. Chem. 58, 14767-14770.

Arunagiri, C., Subashini, A., Saranya, M. \& Thomas Muthiah, P. (2013b). Indian J. Appl. Res. 3, 78-81.

Bruker (2008). APEX2, SAINT and $S A D A B S$. Bruker AXS Inc., Madison, Wisconsin, USA.

Chumakov, Y. M., Tsapkov, V. I., Bocelli, G. \& Antosyak, B. Ya. (2005). Acta Cryst. C61, o460-0463.

Flack, H. D. (1983). Acta Cryst. A39, 876-881.

Sheldrick, G. M. (2008). Acta Cryst. A64, 112-122.

Spek, A. L. (2009). Acta Cryst. D65, 148-155. 


\section{supporting information}

Acta Cryst. (2015). E71, o48 [https://doi.org/10.1107/S2056989014026978]

\section{Crystal structure of 2-\{[(2-chlorophenyl)imino]methyl\}phenol}

\section{Matheswaran Saranya, Annamalai Subashini, Chidambaram Arunagiri and Packianathan Thomas Muthiah}

\section{S1. Comment}

As part of our ongoing studies of Schiff bases (Arunagiri et al., 2013a,b), we now describe the synthesis and structure of the title compound.

An ORTEP view of the asymmetric unit is shown in Figure 1. The asymmetric unit contains a molecule of Schiff base. The compound crystallizes in the orthorhombic space group $P 2_{1} 2_{1} 2_{1}$. The dihedral angle between the salicylidene moiety and amino phenyl plane is $51.42(9)^{\circ}$. The two torsional angles $\tau 1(\mathrm{~N}-\mathrm{C}-\mathrm{C}-\mathrm{C})$ and $\tau 2(\mathrm{C}-\mathrm{N}-\mathrm{C}-\mathrm{C})$ defining the confirmation of the molecule. In the present crystal structure, the torsion angles are $3.2(3)^{\circ}(\mathrm{N} 1-\mathrm{C} 7-\mathrm{C} 8-\mathrm{C} 9)$, $-179.23(2)^{\circ}(\mathrm{N} 1-\mathrm{C} 7-\mathrm{C} 8-\mathrm{C} 13), 47.5(2)^{\circ}(\mathrm{C} 7-\mathrm{N} 1-\mathrm{C} 1-\mathrm{C} 6),-174.48(2)^{\circ}(\mathrm{C} 8-\mathrm{C} 7-\mathrm{N} 1-\mathrm{C} 1)$ and $-135.60(2)^{\circ}$ $(\mathrm{N} 1-\mathrm{C} 7-\mathrm{C} 1-\mathrm{C} 2)$. The $\mathrm{N} 1-\mathrm{C} 7$ distance of 1.275 (2) $\AA$ is normal double bond values and agree well with those observed in other azomethines. The $\mathrm{C} 1-\mathrm{N} 1-\mathrm{C} 7$ bond angle of $118.70(2)^{\circ}$ in the Schiff base ligand has a normal value. The $\mathrm{C} 3-\mathrm{C} 2-\mathrm{C} 1$ angle is $121.15(2)^{\circ}$ is larger than typical hexagonal of $120^{\circ}$. The $\mathrm{C} 8-\mathrm{C} 9-\mathrm{C} 10$ angle is $119.53(2)^{\circ}$ is smaller than typical hexagonal of $120^{\circ}$. This is due to effect of substitution on $\mathrm{Cl} \& \mathrm{OH}$ of the two aromatic rings. The two benzene rings (amino phenyl and salicylaldehyde) and the azomethine group are practically coplanar, as a result of intramolecular $\mathrm{O}-\mathrm{H} \cdots \mathrm{N}\left(\mathrm{O} 1-\mathrm{H} 1 \cdots \mathrm{N} 1\right.$ with bond length of 2.611 (2) $\AA$ and bond angle of $\left.147^{\circ}\right)$ hydrogen bond involving the hydroxy O-atom and azomethine $\mathrm{N}$-atom with graph-set notation $\mathrm{S}(6)$, as shown in Figure 2. Similar intramolecular hydrogen bonds are reported for the crystal structures of 2-(naphthalene-2-yliminomethyl) phenol and $\mathrm{N}$ acetyl-4-[(2-hydroxybenzylidene)-amino]benzenesulfonamide monohydrate (Arunagiri et al., 2013 (a); Chumakov et al., 2005).

\section{S2. Experimental}

An ethanol solution $(25 \mathrm{ml})$ of chlorophenyl amine $(0.25$ mole $)$ was mixed with hydroxy benzaldehyde $(0.25$ mole $)$ and the contents were refluxed for $3 \mathrm{~h}$ and kept aside for crystallization. After a few days a pale yellow colour precipitate was formed. Recrystallization was from $\mathrm{CHCl}_{3} /$ ethanol solution to form yellow needles. FT-IR (KBr pellet) in cm ${ }^{-1}: 3437(\mathrm{O}$ $-\mathrm{H}), 1614 \mathrm{~cm}^{-1}\left(\mathrm{C}=\mathrm{N}\right.$ stretching); ${ }^{1} \mathrm{H}-\mathrm{NMR}\left(400 \mathrm{MHz}, \mathrm{DMSO}-\mathrm{d}^{6}\right)$ in $\delta$ (p.p.m.) 13.17 (s,1H, aromatic $\left.\mathrm{O}-\mathrm{H}\right), 8.63$ (s, $1 \mathrm{H}, \mathrm{C}=\mathrm{N}), 6.93-7.50\left(\mathrm{~m}, 8 H, \mathrm{CH}\right.$ aromatic), ${ }^{13} \mathrm{C}-\mathrm{NMR}\left(400 \mathrm{MHz}, \mathrm{DMSO}-\mathrm{d}^{6}\right)$ in $\delta$ (p.p.m.): $163.3(\mathrm{C}=\mathrm{N}), 161.4$ (phenolic $\mathrm{OH}$ ), Electronic spectrum, $\lambda$ max: 275 and $340 \mathrm{~nm}$ (due to intraligand $\pi-\pi^{*}$ and $\mathrm{n}-\pi^{*}$ transitions); fluorescence spectra, $432 \mathrm{~nm}$ (attributed to the $\mathrm{n}-\pi^{*}$ transition).

\section{S3. Refinement}

All $\mathrm{H}$ atoms were positioned geometrically and treated as riding. The $\mathrm{C}-\mathrm{H}$ and $\mathrm{O}-\mathrm{H}$ bond lengths are $0.93 \AA$ and 0.82 Å respectively. 


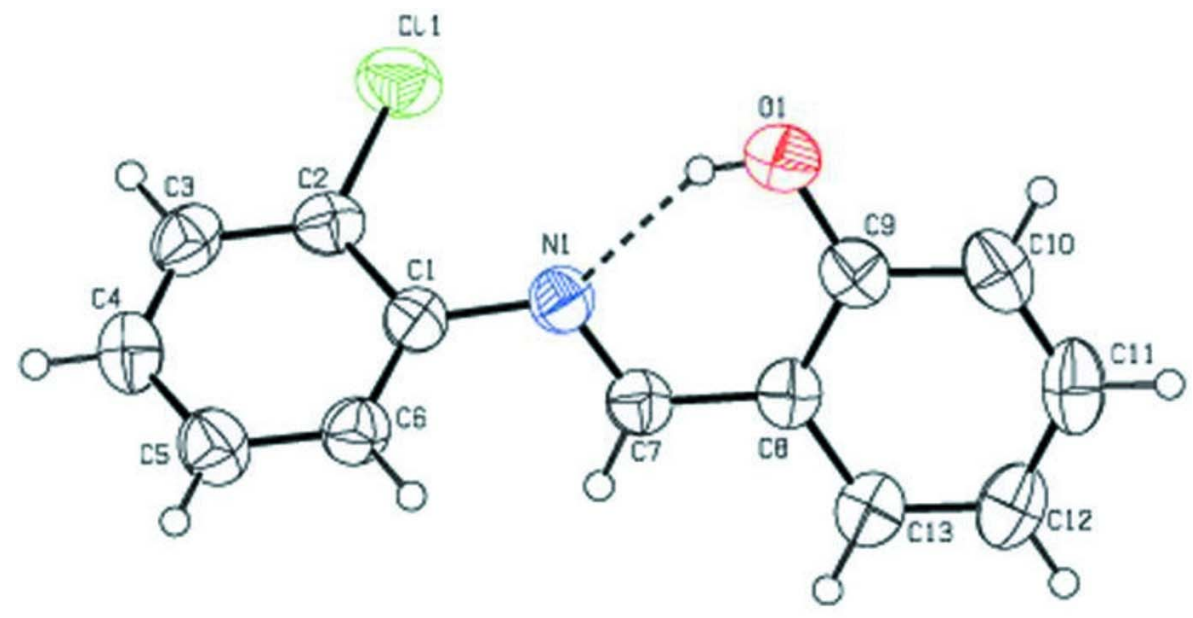

Figure 1

Molecular structure of the title compound with displacement ellipsoids drawn at 50\% probability level. Dashed line indicates intramolecular hydrogen bond.

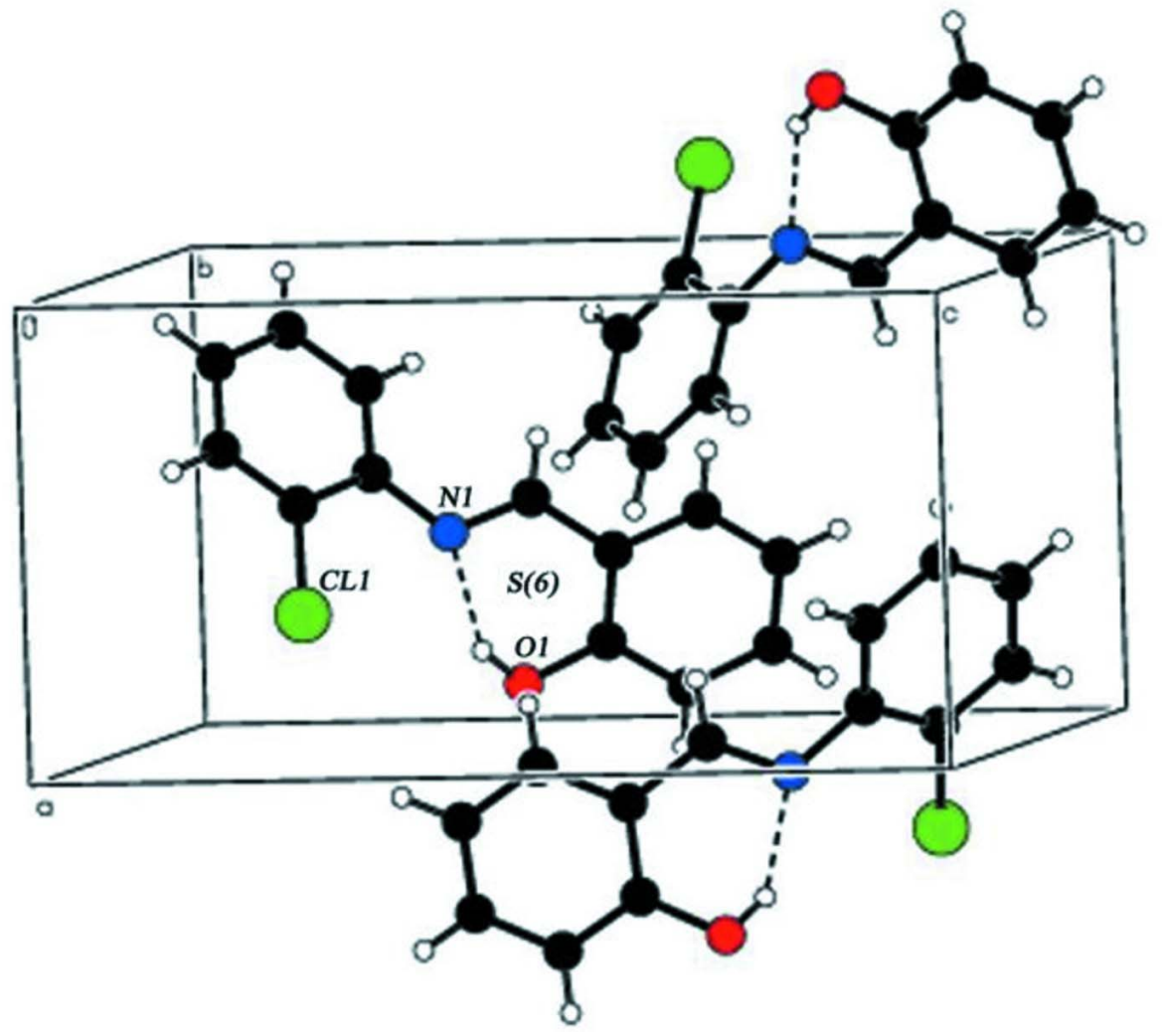

Figure 2

Hydrogen bonding interaction of title compound. 


\section{2-\{[(2-Chlorophenyl)imino]methyl\}phenol}

Crystal data

$\mathrm{C}_{13} \mathrm{H}_{10} \mathrm{ClNO}$

$M_{r}=231.67$

Orthorhombic, $P 2{ }_{1} 2_{1} 2_{1}$

Hall symbol: P 2ac 2ab

$a=6.8591(2) \AA$

$b=12.1829(4) \AA$

$c=13.5405(5) \AA$

$V=1131.50(6) \AA^{3}$

$Z=4$

Data collection

\section{Bruker Kappa APEXII CCD} diffractometer

Radiation source: fine-focus sealed tube

Graphite monochromator

$\omega$ scans

6509 measured reflections

2744 independent reflections

\section{Refinement}

Refinement on $F^{2}$

Least-squares matrix: full

$R\left[F^{2}>2 \sigma\left(F^{2}\right)\right]=0.034$

$w R\left(F^{2}\right)=0.097$

$S=1.06$

2744 reflections

146 parameters

0 restraints

Primary atom site location: structure-invariant direct methods
$F(000)=480$

$D_{\mathrm{x}}=1.360 \mathrm{Mg} \mathrm{m}^{-3}$

Mo $K \alpha$ radiation, $\lambda=0.71073 \AA$

Cell parameters from 45 reflections

$\theta=3.0-28.3^{\circ}$

$\mu=0.31 \mathrm{~mm}^{-1}$

$T=293 \mathrm{~K}$

Cut needle, yellow

$0.30 \times 0.25 \times 0.20 \mathrm{~mm}$

2315 reflections with $I>2 \sigma(I)$

$R_{\text {int }}=0.017$

$\theta_{\max }=28.3^{\circ}, \theta_{\min }=3.0^{\circ}$

$h=-9 \rightarrow 7$

$k=-13 \rightarrow 16$

$l=-18 \rightarrow 10$

Secondary atom site location: difference Fourier map

Hydrogen site location: inferred from

neighbouring sites

$\mathrm{H}$-atom parameters constrained

$w=1 /\left[\sigma^{2}\left(F_{\mathrm{o}}^{2}\right)+(0.0488 P)^{2}+0.0823 P\right]$

where $P=\left(F_{\mathrm{o}}{ }^{2}+2 F_{\mathrm{c}}{ }^{2}\right) / 3$

$(\Delta / \sigma)_{\max }=0.001$

$\Delta \rho_{\max }=0.17 \mathrm{e} \AA^{-3}$

$\Delta \rho_{\min }=-0.17$ e $\AA^{-3}$

Absolute structure: Flack (1983)

Absolute structure parameter: 0.01 (7)

\section{Special details}

Geometry. Bond distances, angles etc. have been calculated using the rounded fractional coordinates. All su's are estimated from the variances of the (full) variance-covariance matrix. The cell e.s.d.'s are taken into account in the estimation of distances, angles and torsion angles

Refinement. Refinement on $F^{2}$ for ALL reflections except those flagged by the user for potential systematic errors.

Weighted $R$-factors $w R$ and all goodnesses of fit $S$ are based on $F^{2}$, conventional $R$-factors $R$ are based on $F$, with $F$ set to zero for negative $F^{2}$. The observed criterion of $F^{2}>\sigma\left(F^{2}\right)$ is used only for calculating - $R$-factor-obs etc. and is not relevant to the choice of reflections for refinement. $R$-factors based on $F^{2}$ are statistically about twice as large as those based on $F$, and $R$-factors based on ALL data will be even larger.

Fractional atomic coordinates and isotropic or equivalent isotropic displacement parameters $\left(\AA^{2}\right)$

\begin{tabular}{lllll}
\hline & $x$ & $y$ & $z$ & $U_{\text {iso }} * U_{\text {eq }}$ \\
\hline C11 & $0.22873(7)$ & $0.87326(5)$ & $0.81891(4)$ & $0.0781(2)$ \\
O1 & $0.41345(19)$ & $0.60666(15)$ & $0.63294(11)$ & $0.0742(5)$ \\
N1 & $0.0830(2)$ & $0.68972(13)$ & $0.69278(10)$ & $0.0531(4)$ \\
C1 & $-0.0473(2)$ & $0.72426(14)$ & $0.76845(12)$ & $0.0486(5)$
\end{tabular}




$\begin{array}{lllll}\text { C2 } & 0.0066(2) & 0.80739(14) & 0.83346(13) & 0.0513(5) \\ \text { C3 } & -0.1161(3) & 0.84060(16) & 0.90860(14) & 0.0606(6) \\ \text { C4 } & -0.2937(3) & 0.78949(18) & 0.92067(14) & 0.0672(7) \\ \text { C5 } & -0.3476(3) & 0.70587(18) & 0.85879(16) & 0.0668(7) \\ \text { C6 } & -0.2258(3) & 0.67361(15) & 0.78292(13) & 0.0563(5) \\ \text { C7 } & 0.0161(2) & 0.67467(14) & 0.60596(13) & 0.0506(5) \\ \text { C8 } & 0.1350(2) & 0.63031(15) & 0.52664(12) & 0.0501(5) \\ \text { C9 } & 0.3282(3) & 0.59651(15) & 0.54312(14) & 0.0556(5) \\ \text { C10 } & 0.4336(3) & 0.54992(17) & 0.46648(17) & 0.0676(7) \\ \text { C11 } & 0.3501(3) & 0.53771(17) & 0.37489(16) & 0.0716(8) \\ \text { C12 } & 0.1616(3) & 0.57133(18) & 0.35676(15) & 0.0701(7) \\ \text { C13 } & 0.0551(3) & 0.61655(16) & 0.43201(13) & 0.0597(6) \\ \text { H1 } & 0.33750 & 0.63660 & 0.67120 & 0.1110^{*} \\ \text { H3 } & -0.07910 & 0.89710 & 0.95080 & 0.0730^{*} \\ \text { H4 } & -0.37710 & 0.81180 & 0.97090 & 0.0810^{*} \\ \text { H5 } & -0.46660 & 0.67070 & 0.86790 & 0.0800^{*} \\ \text { H6 } & -0.26410 & 0.61710 & 0.74100 & 0.0680^{*} \\ \text { H7 } & -0.11310 & 0.69270 & 0.59300 & 0.0610^{*} \\ \text { H10 } & 0.56110 & 0.52680 & 0.47710 & 0.0810^{*} \\ \text { H11 } & 0.42230 & 0.50620 & 0.32410 & 0.0860^{*} \\ \text { H12 } & 0.10740 & 0.56340 & 0.29420 & 0.0840^{*} \\ \text { H13 } & -0.07260 & 0.63860 & 0.42020 & 0.0720^{*}\end{array}$

Atomic displacement parameters $\left(\AA^{2}\right)$

\begin{tabular}{lllllll}
\hline & $U^{11}$ & $U^{22}$ & $U^{33}$ & $U^{12}$ & $U^{13}$ & $U^{23}$ \\
\hline C11 & $0.0627(3)$ & $0.0739(3)$ & $0.0977(4)$ & $-0.0117(2)$ & $-0.0077(2)$ & $-0.0088(3)$ \\
O1 & $0.0569(7)$ & $0.0894(11)$ & $0.0762(9)$ & $0.0072(8)$ & $-0.0098(6)$ & $-0.0086(8)$ \\
N1 & $0.0541(7)$ & $0.0507(8)$ & $0.0545(7)$ & $0.0050(6)$ & $-0.0011(6)$ & $-0.0023(6)$ \\
C1 & $0.0520(9)$ & $0.0442(9)$ & $0.0496(8)$ & $0.0093(7)$ & $-0.0039(7)$ & $0.0014(7)$ \\
C2 & $0.0536(8)$ & $0.0423(8)$ & $0.0579(9)$ & $0.0038(7)$ & $-0.0103(7)$ & $0.0030(7)$ \\
C3 & $0.0738(12)$ & $0.0493(10)$ & $0.0588(10)$ & $0.0110(8)$ & $-0.0099(8)$ & $-0.0079(8)$ \\
C4 & $0.0715(12)$ & $0.0656(12)$ & $0.0644(11)$ & $0.0088(10)$ & $0.0122(9)$ & $-0.0050(10)$ \\
C5 & $0.0633(10)$ & $0.0610(12)$ & $0.0761(12)$ & $-0.0017(9)$ & $0.0086(9)$ & $-0.0019(10)$ \\
C6 & $0.0597(9)$ & $0.0477(9)$ & $0.0614(9)$ & $0.0011(8)$ & $-0.0033(8)$ & $-0.0063(8)$ \\
C7 & $0.0516(8)$ & $0.0436(8)$ & $0.0566(9)$ & $0.0050(7)$ & $-0.0025(7)$ & $0.0009(7)$ \\
C8 & $0.0578(8)$ & $0.0392(8)$ & $0.0533(9)$ & $-0.0004(7)$ & $0.0033(7)$ & $0.0024(7)$ \\
C9 & $0.0547(8)$ & $0.0453(9)$ & $0.0667(10)$ & $-0.0036(7)$ & $0.0040(8)$ & $0.0034(8)$ \\
C10 & $0.0609(10)$ & $0.0551(11)$ & $0.0869(14)$ & $0.0012(9)$ & $0.0190(10)$ & $0.0028(10)$ \\
C11 & $0.0956(15)$ & $0.0490(11)$ & $0.0703(13)$ & $0.0000(11)$ & $0.0317(11)$ & $-0.0017(9)$ \\
C12 & $0.0957(14)$ & $0.0612(12)$ & $0.0533(10)$ & $-0.0001(12)$ & $0.0053(10)$ & $-0.0001(9)$ \\
C13 & $0.0702(10)$ & $0.0531(10)$ & $0.0559(9)$ & $0.0019(9)$ & $0.0001(8)$ & $0.0025(8)$ \\
& & & & & & \\
\hline
\end{tabular}

Geometric parameters $\left(\AA,{ }^{\circ}\right)$

\begin{tabular}{llll}
\hline $\mathrm{C} 11-\mathrm{C} 2$ & $1.7333(15)$ & $\mathrm{C} 9-\mathrm{C} 10$ & $1.386(3)$ \\
$\mathrm{O} 1-\mathrm{C} 9$ & $1.355(2)$ & $\mathrm{C} 10-\mathrm{C} 11$ & $1.374(3)$ \\
$\mathrm{O} 1-\mathrm{H} 1$ & 0.8200 & $\mathrm{C} 11-\mathrm{C} 12$ & $1.378(3)$
\end{tabular}




\begin{tabular}{|c|c|c|c|}
\hline $\mathrm{N} 1-\mathrm{C} 7$ & $1.275(2)$ & $\mathrm{C} 12-\mathrm{C} 13$ & $1.369(3)$ \\
\hline $\mathrm{N} 1-\mathrm{C} 1$ & $1.423(2)$ & $\mathrm{C} 3-\mathrm{H} 3$ & 0.9300 \\
\hline $\mathrm{C} 1-\mathrm{C} 6$ & $1.385(2)$ & $\mathrm{C} 4-\mathrm{H} 4$ & 0.9300 \\
\hline $\mathrm{C} 1-\mathrm{C} 2$ & $1.392(2)$ & $\mathrm{C} 5-\mathrm{H} 5$ & 0.9300 \\
\hline $\mathrm{C} 2-\mathrm{C} 3$ & $1.381(3)$ & $\mathrm{C} 6-\mathrm{H} 6$ & 0.9300 \\
\hline $\mathrm{C} 3-\mathrm{C} 4$ & $1.378(3)$ & $\mathrm{C} 7-\mathrm{H} 7$ & 0.9300 \\
\hline $\mathrm{C} 4-\mathrm{C} 5$ & $1.370(3)$ & $\mathrm{C} 10-\mathrm{H} 10$ & 0.9300 \\
\hline $\mathrm{C} 5-\mathrm{C} 6$ & $1.381(3)$ & C11-H11 & 0.9300 \\
\hline $\mathrm{C} 7-\mathrm{C} 8$ & $1.453(2)$ & $\mathrm{C} 12-\mathrm{H} 12$ & 0.9300 \\
\hline $\mathrm{C} 8-\mathrm{C} 13$ & $1.404(2)$ & $\mathrm{C} 13-\mathrm{H} 13$ & 0.9300 \\
\hline $\mathrm{C} 8-\mathrm{C} 9$ & $1.406(2)$ & & \\
\hline $\mathrm{C} 9-\mathrm{O} 1-\mathrm{H} 1$ & 109.00 & $\mathrm{C} 11-\mathrm{C} 12-\mathrm{C} 13$ & $119.17(19)$ \\
\hline $\mathrm{C} 1-\mathrm{N} 1-\mathrm{C} 7$ & $118.70(13)$ & $\mathrm{C} 8-\mathrm{C} 13-\mathrm{C} 12$ & $121.26(18)$ \\
\hline $\mathrm{N} 1-\mathrm{C} 1-\mathrm{C} 6$ & $121.68(15)$ & $\mathrm{C} 2-\mathrm{C} 3-\mathrm{H} 3$ & 120.00 \\
\hline $\mathrm{C} 2-\mathrm{C} 1-\mathrm{C} 6$ & $118.00(15)$ & $\mathrm{C} 4-\mathrm{C} 3-\mathrm{H} 3$ & 120.00 \\
\hline $\mathrm{N} 1-\mathrm{C} 1-\mathrm{C} 2$ & $120.24(13)$ & $\mathrm{C} 3-\mathrm{C} 4-\mathrm{H} 4$ & 120.00 \\
\hline $\mathrm{C} 11-\mathrm{C} 2-\mathrm{C} 3$ & $118.93(14)$ & $\mathrm{C} 5-\mathrm{C} 4-\mathrm{H} 4$ & 120.00 \\
\hline $\mathrm{C} 1-\mathrm{C} 2-\mathrm{C} 3$ & $121.15(15)$ & $\mathrm{C} 4-\mathrm{C} 5-\mathrm{H} 5$ & 120.00 \\
\hline $\mathrm{C} 11-\mathrm{C} 2-\mathrm{C} 1$ & $119.90(12)$ & $\mathrm{C} 6-\mathrm{C} 5-\mathrm{H} 5$ & 120.00 \\
\hline $\mathrm{C} 2-\mathrm{C} 3-\mathrm{C} 4$ & $119.59(17)$ & $\mathrm{C} 1-\mathrm{C} 6-\mathrm{H} 6$ & 120.00 \\
\hline $\mathrm{C} 3-\mathrm{C} 4-\mathrm{C} 5$ & $120.14(19)$ & $\mathrm{C} 5-\mathrm{C} 6-\mathrm{H} 6$ & 120.00 \\
\hline $\mathrm{C} 4-\mathrm{C} 5-\mathrm{C} 6$ & $120.22(19)$ & $\mathrm{N} 1-\mathrm{C} 7-\mathrm{H} 7$ & 119.00 \\
\hline $\mathrm{C} 1-\mathrm{C} 6-\mathrm{C} 5$ & $120.87(17)$ & $\mathrm{C} 8-\mathrm{C} 7-\mathrm{H} 7$ & 119.00 \\
\hline $\mathrm{N} 1-\mathrm{C} 7-\mathrm{C} 8$ & $122.21(13)$ & $\mathrm{C} 9-\mathrm{C} 10-\mathrm{H} 10$ & 120.00 \\
\hline $\mathrm{C} 7-\mathrm{C} 8-\mathrm{C} 13$ & $120.01(14)$ & $\mathrm{C} 11-\mathrm{C} 10-\mathrm{H} 10$ & 120.00 \\
\hline $\mathrm{C} 9-\mathrm{C} 8-\mathrm{C} 13$ & $118.56(16)$ & $\mathrm{C} 10-\mathrm{C} 11-\mathrm{H} 11$ & 119.00 \\
\hline $\mathrm{C} 7-\mathrm{C} 8-\mathrm{C} 9$ & $121.39(15)$ & $\mathrm{C} 12-\mathrm{C} 11-\mathrm{H} 11$ & 119.00 \\
\hline $\mathrm{O} 1-\mathrm{C} 9-\mathrm{C} 10$ & $118.96(18)$ & $\mathrm{C} 11-\mathrm{C} 12-\mathrm{H} 12$ & 120.00 \\
\hline $\mathrm{C} 8-\mathrm{C} 9-\mathrm{C} 10$ & $119.53(18)$ & $\mathrm{C} 13-\mathrm{C} 12-\mathrm{H} 12$ & 120.00 \\
\hline $\mathrm{O} 1-\mathrm{C} 9-\mathrm{C} 8$ & $121.51(17)$ & $\mathrm{C} 8-\mathrm{C} 13-\mathrm{H} 13$ & 119.00 \\
\hline $\mathrm{C} 9-\mathrm{C} 10-\mathrm{C} 11$ & $120.17(19)$ & $\mathrm{C} 12-\mathrm{C} 13-\mathrm{H} 13$ & 119.00 \\
\hline $\mathrm{C} 10-\mathrm{C} 11-\mathrm{C} 12$ & $121.3(2)$ & & \\
\hline $\mathrm{C} 7-\mathrm{N} 1-\mathrm{C} 1-\mathrm{C} 2$ & $-135.60(17)$ & $\mathrm{N} 1-\mathrm{C} 7-\mathrm{C} 8-\mathrm{C} 9$ & $3.2(3)$ \\
\hline $\mathrm{C} 7-\mathrm{N} 1-\mathrm{C} 1-\mathrm{C} 6$ & $47.5(2)$ & $\mathrm{N} 1-\mathrm{C} 7-\mathrm{C} 8-\mathrm{C} 13$ & $-179.23(17)$ \\
\hline $\mathrm{C} 1-\mathrm{N} 1-\mathrm{C} 7-\mathrm{C} 8$ & $-174.48(16)$ & $\mathrm{C} 7-\mathrm{C} 8-\mathrm{C} 9-\mathrm{O} 1$ & $-1.9(3)$ \\
\hline $\mathrm{N} 1-\mathrm{C} 1-\mathrm{C} 2-\mathrm{Cl} 1$ & $2.9(2)$ & $\mathrm{C} 7-\mathrm{C} 8-\mathrm{C} 9-\mathrm{C} 10$ & $177.01(17)$ \\
\hline $\mathrm{C} 6-\mathrm{C} 1-\mathrm{C} 2-\mathrm{Cl} 1$ & $179.83(13)$ & $\mathrm{C} 13-\mathrm{C} 8-\mathrm{C} 9-\mathrm{O} 1$ & $-179.45(18)$ \\
\hline $\mathrm{C} 6-\mathrm{C} 1-\mathrm{C} 2-\mathrm{C} 3$ & $-1.7(3)$ & $\mathrm{C} 13-\mathrm{C} 8-\mathrm{C} 9-\mathrm{C} 10$ & $-0.6(3)$ \\
\hline $\mathrm{N} 1-\mathrm{C} 1-\mathrm{C} 2-\mathrm{C} 3$ & $-178.70(16)$ & $\mathrm{C} 7-\mathrm{C} 8-\mathrm{C} 13-\mathrm{C} 12$ & $-177.66(18)$ \\
\hline $\mathrm{C} 2-\mathrm{C} 1-\mathrm{C} 6-\mathrm{C} 5$ & $0.9(3)$ & $\mathrm{C} 9-\mathrm{C} 8-\mathrm{C} 13-\mathrm{C} 12$ & $0.0(3)$ \\
\hline $\mathrm{N} 1-\mathrm{C} 1-\mathrm{C} 6-\mathrm{C} 5$ & $177.85(17)$ & $\mathrm{O} 1-\mathrm{C} 9-\mathrm{C} 10-\mathrm{C} 11$ & $179.40(19)$ \\
\hline $\mathrm{C} 11-\mathrm{C} 2-\mathrm{C} 3-\mathrm{C} 4$ & $179.58(15)$ & $\mathrm{C} 8-\mathrm{C} 9-\mathrm{C} 10-\mathrm{C} 11$ & $0.5(3)$ \\
\hline $\mathrm{C} 1-\mathrm{C} 2-\mathrm{C} 3-\mathrm{C} 4$ & $1.1(3)$ & $\mathrm{C} 9-\mathrm{C} 10-\mathrm{C} 11-\mathrm{C} 12$ & $0.2(3)$ \\
\hline $\mathrm{C} 2-\mathrm{C} 3-\mathrm{C} 4-\mathrm{C} 5$ & $0.3(3)$ & $\mathrm{C} 10-\mathrm{C} 11-\mathrm{C} 12-\mathrm{C} 13$ & $-0.8(3)$ \\
\hline $\mathrm{C} 3-\mathrm{C} 4-\mathrm{C} 5-\mathrm{C} 6$ & $-1.1(3)$ & $\mathrm{C} 11-\mathrm{C} 12-\mathrm{C} 13-\mathrm{C} 8$ & $0.7(3)$ \\
\hline $\mathrm{C} 4-\mathrm{C} 5-\mathrm{C} 6-\mathrm{C} 1$ & $0.5(3)$ & & \\
\hline
\end{tabular}


Hydrogen-bond geometry $\left(A,{ }^{\circ}\right)$

\begin{tabular}{lllll}
\hline$D-\mathrm{H} \cdots A$ & $D-\mathrm{H}$ & $\mathrm{H} \cdots A$ & $D \cdots A$ & $D-\mathrm{H} \cdots A$ \\
\hline $\mathrm{O} 1-\mathrm{H} 1 \cdots \mathrm{N} 1$ & 0.82 & 1.88 & $2.611(2)$ & 147 \\
\hline
\end{tabular}

